\title{
Operationalizing Cooperative Approaches Under the Paris Agreement by Valuing Mitigation Outcomes
}

\begin{abstract}
Justin D Macinante*
The Paris Agreement recognises the heterogeneity of approaches being implemented across jurisdictions and that parties may voluntarily engage in cooperative approaches involving use of internationally transferred mitigation outcomes towards their nationally determined contributions. Many of these diverse approaches involve putting a price on carbon, usually through emissions trading schemes or carbon taxes. Engaging the private sector at scale will be crucial for these carbon pricing mechanisms to operate efficiently in bringing about behavioural changes that will accelerate mitigation of greenhouse gas emissions and generate greater investment in climate change solutions. This will only happen on the scale necessary if there are clear, well-designed and properly functioning markets for the international transfer of mitigation outcomes and for trading carbon assets more generally. Achieving this mandates the development and implementation of processes to value mitigation outcomes, that is, to assess their mitigation value, and corresponding institutional arrangements to oversee such assessment processes. Mitigation value assessments will facilitate fungibility, enabling trading of these carbon assets across jurisdictions. This paper begins with a look at how the inter-governmental negotiations are addressing this need, and analyses how the literature has approached the subject, before setting out proposals aimed at stimulating debate on what these processes and institutional arrangements might appropriately include.
\end{abstract}

\section{Introduction}

The Kyoto Protocol took about seven years to operationalize and about another seven to reach the end of the underwhelming first commitment period. The European Union and Switzerland have taken seven years to reach agreement on linking their respective emission trading schemes. ${ }^{1}$

The proceedings of the $48^{\text {th }}$ meeting of the Subsidiary Body for Scientific and Technical Advice (SBSTA48) in Bonn in May 2018, where guidance on Cooperative Approaches referred to in Article 6.2 of the Paris Agreement was to be discussed, suggest seven years would appear to be a wildly optimistic expectation for operationalization of this provision. An inordinate amount of time seemed to be spent discussing the meeting process and how it should proceed, judging from both the SBSTA conclusions ${ }^{2}$ and the dearth of clear positions and lack of progress in the course of discussions. This was evident in a revised informal note, containing draft elements of the guidance, offered by the co-chairs as a work in progress, not representing consensus, but to support Parties in the negotiations. ${ }^{3}$

It was resolved to reconvene SBSTA48 in Thailand in September 2018. Paragraph 36 of the Decisions

DOI: $10.21552 / \mathrm{cclr} / 2018 / 3 / 12$

* *BSc LLB (UNSW) MEL (Hon) (Syd.); Policy Lead, DLT4NCM project, University of Edinburgh. The author thanks Dr. Matthew Brander and Prof. Michael Mehling for their helpful comments. Errors and omissions remain solely those of the author. For correspondence: <J.D.Macinante@ed.ac.uk>

1 EU Commission, 'EU and Switzerland sign agreement to link emissions trading systems' (23 November 2017) <https://ec .europa.eu/clima/news/eu-and-switzerland-sign-agreement-link -emissions-trading-systems_en> accessed 31 August 2018.

2 International Institute for Sustainable Development, 'Earth Negotiations Bulletin - Summary of Bonn Climate Change Conference' (2018) 12, 726 .

3 SBSTA 48, 'Agenda item 12(a) Revised informal note containing draft elements of the guidance on cooperative approaches referred to in Article 6, paragraph 2, of the Paris Agreement' (10 May 2018) <https://unfccc.int/sites/default/files/resource/SBSTA \%2048_IN_12a\%20Art\%206\%20para\%202.pdf> accessed 15 May 2018. 
adopted by the Conference of the Parties in Paris ${ }^{4}$ requests the SBSTA develop and recommend this guidance for adoption by the Conference of Parties serving as the meeting of the Parties to the Paris Agreement at its first session (COP24/CMA1), that is, by December 2018. It seems unlikely that the SBSTA will be able to provide a sufficient level of detail by CMA1 in response to that request. The question is by when such guidance might be ready?

At SBSTA47, to facilitate deliberations at SBSTA48, the Chair had been requested to prepare an informal document containing the elements of guidance on cooperative approaches, based on prior submissions by Parties (over the preceding four negotiating sessions). ${ }^{5}$ Section VI of the revised informal note offered by the co-chairs during SBSTA $48^{6}$ addresses internationally transferred mitigation outcomes (ITMOs). Sub-section A deals with ITMOs that can be used towards NDCs, with paragraph headings covering who has responsibility for determining which ones can be so used; measurement; form; and scope. Sub-section B covers ITMO characteristics and sub-section $\mathrm{C}$ covers 'other ITMOs'. But there isn't much flesh on these bones.

In relation to measurement, for instance, the list of potential options in the note includes that an ITMO might be (a) equal to one metric tonne of carbon dioxide equivalent $\left(\mathrm{CO}_{2}-\mathrm{e}\right)$; (b) measured in a metric

4 United Nations, 'Report of the Conference of the Parties on its twenty-first session, held in Paris from 30 November to 13 December 2015' (29 January 2016) FCCC/CP/2015/10/Add.1.

5 SBSTA.48.Informal.2, Informal document containing the draft elements of guidance on cooperative approaches referred to in Article 6, paragraph 2, of the Paris Agreement, Informal document by the chair', Para 2, Mandate (16 March 2018)

6 (n 3).

7 ibid 6 .

8 The World Bank has reported, for instance, that approximately one hundred Parties to the Paris Agreement, accounting for $58 \%$ of global GHG emissions, have indicated they are planning or considering use of carbon pricing. These include three of the world's largest emitters, in China, India and Brazil. See, World Bank Group, 'State and Trends of Carbon Pricing 2016' (2016) 22.

9 Jurisdiction for these purposes would normally conform with the usual (English) language sense of the term, that is a geographical unit in the form of a 'state', and the assumption is that any ETS or other pricing mechanism is based on a legislative-administrative framework. A jurisdiction, though, could be, for instance, a state/province/region within a (quasi-) federal system, just as much as a unitary or federal (national) state. Depending on the nature of the ETS or pricing mechanism there may be other possible variations, e.g., one established by a (large) metropolitan unit, or one in which a coalition of sovereign states has its own fully integrated (and thus 'internal') ETS (as presently within the EU). other than tonnes $\mathrm{CO}_{2}-\mathrm{e}$; (c) measured in both; or (d) greenhouse gases (GHGs) and non-GHGs. It provides also that an ITMO might be calculated (a) in accordance with methodologies and common metrics assessed by the IPCC and adopted by the COP/CMA; or (b) using global warming potentials assessed/recommended by the IPCC and adopted by the COP/CMA; or (c) determined by the Parties implementing the cooperative approach. ${ }^{7}$ These elements, reflecting viewpoints put to the co-chairs by Parties, give the appearance of simply passing the onus to others; at the same time, the apparent brevity of consideration given to this aspect is disquieting. Absence of any mention of ITMO 'mitigation value' seems to be an oversight, or worse, a deliberate skirting around what is a difficult, but fundamental issue. It even leaves open a continuation of Kyoto Protocol thinking that by defining units to be of a certain value, they will be that value.

There is no doubt that a physical tonne of carbon dioxide equivalent greenhouse gas $\left(\mathrm{CO}_{2}-\mathrm{e}\right)$ emitted in one part of the globe will be the same as one emitted somewhere else on the globe. Similarly, a physical tonne of $\mathrm{CO}_{2}$-e mitigated in one part of the globe will be the same as one mitigated somewhere else on the globe. However, an action to bring about that mitigation outcome in one part of the globe will not necessarily be the same, nor achieve the same outcome, as an action in another part of the globe: simply defining a unit of mitigation outcome from both actions as being equal in measured value (that is, for instance, both being equal to one metric tonne $\mathrm{CO}_{2}$-e) does not change this fact. The Paris Agreement recognises this heterogeneity of mitigation outcomes.

Engaging the private sector at scale is crucial if carbon pricing mechanisms, which appear to be the mitigation action of choice for an increasing number of jurisdictions, ${ }^{8}$ are to operate efficiently in bringing about behavioural changes that will accelerate mitigation of GHG emissions and generate greater investment in climate change solutions. This will only happen on the scale necessary if there are clear, well-designed and properly functioning markets for the international transfers of mitigation outcomes and for trading carbon assets generally. For these markets to exist, a common metric is necessary to enable comparability of these heterogeneous mitigation outcomes and fungibility across jurisdictions. ${ }^{9}$

It should go without saying that, while these markets - if well-designed - should operate with as little 
intervention as possible to maximise their efficiency and effectiveness, that needs to happen within an equally well-designed boundary framework of climate change rules. Processes to value mitigation outcomes, that is, to assess the mitigation value of mitigation actions not just by Paris Agreement parties, but by regional, sub-national and even municipal jurisdictions, ${ }^{10}$ and the institutional arrangements to oversee those processes, will be fundamental to both well-designed markets and well-designed climate change rules.

This paper analyses what might be included in those processes and commensurate institutional arrangements, with a view to stimulating a deeper, constructive debate on guidance on cooperative approaches under Article 6.2. It begins in Section II by considering what mitigation value is and suggesting an approach as to how mitigation value might be assessed, as a way of stimulating debate on this important subject. Section III reviews academic literature on the subject of comparative evaluations in the context of climate change policy and some specific approaches that are developing, or could be adapted into, methodologies for determining the value of mitigation outcomes. Section IV looks at institutional arrangements, as well as outlining possible options for such institutions in future climate policy architecture. The paper concludes by considering how such an approach to operationalising cooperative approaches under Article 6 might be taken forward, given the increasingly pressing need for effective mitigation action.

\section{Mitigation Value and Mitigation Value Assessment}

Consider a transaction in which an entity authorised by Jurisdiction 1 transfers a mitigation outcome to an entity in Jurisdiction 2. The mitigation outcome is a unit (a carbon unit or 'CU') from Jurisdiction 1's emission trading scheme $\left(\mathrm{ETS}_{1}\right)$ and is going to Jurisdiction 2's emission trading scheme $\left(\mathrm{ETS}_{2}\right)$. The unit, $\mathrm{CU}_{1}$, has an entitlement in ETS $\mathrm{S}_{1}$ of one tonne $\mathrm{CO}_{2}-\mathrm{e}$, that is, it entitles the holder to surrender it in ETS in order to acquit a compliance obligation under that scheme in respect of the emission (by the holder) of one tonne $\mathrm{CO}_{2}$-e.

The transfer raises a number of questions, but to consider just two: what is the 'value' of $\mathrm{CU}_{1}$ (whether in $\mathrm{ETS}_{1}$ or $\mathrm{ETS}_{2}$ ) and is it the same as the entitlement (that is, one tonne $\mathrm{CO}_{2}-\mathrm{e}$ )?

Since the purpose of the ETS is to mitigate emissions, it would seem reasonable to consider the value to be the 'mitigation value' of the unit. While there is no widely accepted formal definition for such at present, mitigation value (MV) might be described here in terms of the physical amount of reduced or avoided GHG emission to, or GHG sequestered from, the atmosphere, which can be attributed to a particular tradable unit (mitigation outcome) under, or derived from, a GHG mitigation scheme (mitigation action). Thus, in the transaction considered here, the value of $\mathrm{CU}_{1}$ will be $M V_{1}$; but what is $\mathrm{MV}_{1}$ ?

The answer proposed by this paper, for discussion, is that $\mathrm{MV}_{1}$ (or, for that matter, the value of any allowance unit issued in any ETS), in the first instance, will be equal to the area between the business-as-usual (BAU) emission curve and the ETS-achieved emissions curve, divided by the number of CUs issued in the ETS, in the relevant time period (e.g., per annum)..$^{11}$ The value CUs represent is not the area under the emission reduction curve, which rather represents the amount of emissions permitted under the scheme's cap, that is, CUs available for the domestic accounting purpose of reconciliation against actual emissions under that ETS; whereas, on the other hand, the mitigation value of those CUs is the area between that curve and the business-as-usual (BAU) curve above it: in other words, the difference in emissions with and without the scheme. This represents the amount of emissions reduced by the ETS in the relevant time period, if entities under that scheme have complied. ${ }^{12}$

All the same, to arrive at an accurate MV, this primary amount of value needs to be adjusted to take account of: ${ }^{13}$

10 Although for the purposes of the discussion in this paper, consideration of mitigation value and its assessment is couched in terms of transfers between Paris Agreement parties.

11 The marginal impact of the ETS, rather than this 'averaged' value, may also be an appropriate starting point: this paper does not offer a definitive view, but rather raises this as a question for debate.

12 See also Justin D Macinante, 'From Homogeneity to Heterogeneity and the Fundamental Question - What Is Being Traded?' (2017) Edinburgh School of Law Research Paper No 2017/15.

13 These factors are set out in the World Bank's Networked Carbon Markets initiative, see, The World Bank, 'Brief: Networked Carbon Markets' (2017) <http://www.worldbank.org/en/topic/ climatechange/brief/globally-networked-carbon-markets $>$ accessed 31 August 2018. 
(i) risks relating to the characteristics of that particular scheme (for example, the reliability of the monitoring, reporting and verification (MRV) undertaken); and

(ii) risks relating to the characteristics of the jurisdiction's overall suite of mitigation policies (for example, overall coverage, gaps in emissions covered, overlaps and leakage across the suite of measures).

In the context of overall climate change policy, the primary value might be adjusted also to take account of the characteristics of the jurisdiction's contribution to addressing global climate change (for instance, the ambition demonstrated by its NDC). Adjustment along these lines would involve broader, more political than purely physical, considerations.

Returning to the example transaction, if, in ETS ${ }_{1}$, the entitlement nominal value of one tonne $\mathrm{CO}_{2}$-e for $\mathrm{CU}_{1}$ is based on the area under the ETS-achieved emissions curve, then it is likely that $M V_{1}$ will be less than one tonne $\mathrm{CO}_{2}$-e. ${ }^{14}$ How much less will depend:

- firstly, on the relative size of (a) the area between the BAU curve and ETS-achieved emissions curve, to (b) the area under the ETS-achieved emissions curve, and

- secondly, on adjustments for the risks mentioned above.

Thus, the MV of a unit from an ETS (mitigation action) is not the same as the emission entitlement of that unit (e.g., nominally one tonne $\mathrm{CO}_{2}$-e) in the ETS. Does this matter if the unit becomes an ITMO? $\mathrm{Ab}$ solutely. Again, in the example: will $\mathrm{CU}_{1}$ still have $\mathrm{MV}_{1}$ when it moves to ETS 2 ? Yes, since the MV is the physical mitigation attributable to that particular unit. A further question, however, is whether $\mathrm{CU}_{1}$ will

14 That is, of course, unless the ETS has resulted in more than halving of emissions, which is probably unlikely in most instances.

15 SBSTA, 'Informal note by the co-chairs, Third iteration, 12 November 2017, Subsidiary Body for Scientific and technological Advice, Forty-Seventh meeting' (November 2017) <http://unfccc .int/files/meetings/bonn_nov_2017/in-session/application/pdf/ sbsta47_11a_third_informal_note_.pdf $>$ accessed 27 August 18.

16 This would be difficult to do unless the mitigation value of units cancelled is known.

17 Interestingly also, that in relation to infrastructure, both 'blockchain' and 'a centrally accessible distributed ledger' have been mentioned.

18 World Bank Group, 'State and Trends of Carbon Pricing 2017' (2017) Annex III. still be $\mathrm{CU}_{1}$ when it is transferred or traded? When $\mathrm{CU}_{1}$ moves into $\mathrm{ETS}_{2}$, it may be administratively more feasible and simpler for it to be converted into the domestic equivalent, that is, a $\mathrm{CU}_{2}$. However, $\mathrm{CU}_{2} \mathrm{~s}$ are likely to have a different $\mathrm{MV}$ to $\mathrm{CU}_{1} \mathrm{~s}$ because:

- both the BAU emissions curve and the ETSachieved emissions curve in ETS $_{2}$ will differ from that in $\mathrm{ETS}_{1}$; and

- the risks mentioned above will be different, hence so will be the adjustments to $\mathrm{CU}_{2} \mathrm{~s}$.

At present, differences in design, implementation and standards detract from the effectiveness of the diverse and heterogeneous carbon pricing mechanisms in various jurisdictions. While this reflects local preferences, it means the market will remain fragmented until there is a mechanism to convert, enabling comparability and thus fungibility of the units across schemes. Determining the MV of the units provides such a mechanism.

Reports of the negotiations concerning guidance on cooperative approaches referred to in Article 6.2, Paris Agreement, ${ }^{15}$ indicate discussions have touched on issues that might be relevant to mitigation value, such as:

- in relation to environmental integrity, the quality of units;

- in relation to governance oversight arrangements, third party technical review of the environmental integrity of ITMOs created/approval of ITMOs;

- in relation to governance role of the secretariat, reporting on overall mitigation of global emissions delivered through cancellation/discounting; ${ }^{16}$

- in relation to reporting on use of ITMOs, information including characteristics of units, originating programmes, source of ITMOs, vintage/time periods of ITMOs. ${ }^{17}$

It is noted also that there has been consensus in the negotiations on the need for common accounting standards and transaction procedures, and for quantifying ITMOs (with the possibility of tonnes $\mathrm{CO}_{2}-\mathrm{e}$ as a standard unit mentioned).${ }^{18}$ While it is hoped these points will crystallise as elements of a coherent set of guidance measures, or at least principles, that embody the concept of mitigation value of outcomes by $\mathrm{COP} 24$, on present indications that seems unlikely. 


\section{Comparative Evaluations in the Context of Climate Change Policy}

Given its significance to the outcome of the negotiations, it is surprising how little discussion there has been of mitigation value, or the valuing of emission trading scheme units, in the literature. One reason for this might be the fact that, to date, the majority of studies on trading between schemes seem to have focused on full bilateral linking under which the units are homogenised so as to fully fungible in all participating systems. ${ }^{19}$ Another possible explanation might be the historical fact of the homogeneous approach taken prior to the Paris Agreement, that is, under the Kyoto Protocol, where the value of all traded units was simply defined as being equal to one tonne $\mathrm{CO}_{2}$-e. Moreover, the literature has focused on trying to define what the units are in a legal sense (for example, property right, commodity and so on) and what they entitle the holder to do, rather than on what their value might be. ${ }^{20}$

All the same, there is analysis of MV in the literature, most conspicuously by Joseph Aldy and colleagues. ${ }^{21}$ In anticipation of outcomes under the Paris Agreement, Aldy ${ }^{22}$ noted that assessments of mitigation value could play an important role in linking between countries with disparate mitigation policies. These MV assessments, it was speculated, could inform the linking agreement through exchange rates which, if transparent, could be used to incentivise higher ambition on the part of more poorly performing jurisdictions. ${ }^{23}$

More recently, in discussing the World Bank Networked Carbon Markets (NCM) initiative (a fundamental tenet of which is an independent assessment framework for determining the climate change mitigation value of actions), another commentator, Michael Mehling, observes that while a move from a regime based on compatibility of systems and equivalence of traded units, to one that seeks to quantify and compare mitigation effort, offers interesting perspectives, it will also give rise to political controversy and raise similar challenges to those experienced in negotiations to date. ${ }^{24}$ While it is inevitable perhaps that any proposed change to the status quo meets resistance, it could be observed in response firstly, that many of the challenges experienced in negotiations to date have not yet gone away and change such as that proposed might be just what is needed to cut the Gordian Knot that these challenges contin- ue to pose. Secondly, it is observed also that the Paris Agreement has already begun the move away from a regime based on compatibility of systems and equivalence of traded units. Thus, the Paris Agreement parties, themselves, have initiated the process. It is just that the detail of that shift is taking a long time - perhaps too long - to tease out into properly workable operational measures.

But to consider Mehling's point further, it could be said that the MV approach simply shifts the political barriers from agreeing, for example, system harmonisation under a linking or similar arrangement, to agreeing a platform that quantifies and rates respective jurisdictions' efforts (or, at least, the outcomes of those efforts). Two lines of argument are proffered in response.

First, in the context of heterogeneous mitigation efforts recognised by the Paris Agreement, the application of analytical tools and corresponding means for data gathering, is crucial 'for assessing the country-level, comparative, and aggregate impacts of those efforts. ${ }^{25}$ These tools and associated data rely

19 Aki Kachi et al, 'Linking Emissions Trading Systems: A Summary of Current Research' (January 2015) International Carbon Action Partnership Report, 11.

20 (n 12)

21 Joseph E Aldy, 'Designing a Bretton Woods Institution to Address Climate Change' (2012) HKS Faculty Research Working Paper Series RWP12-017; Joseph E Aldy, 'The crucial role of policy surveillance in international climate policy' (2014) 126 Climatic Change 3 - 4, 279 - 292; Joseph E Aldy and William A Pizer, 'Comparing emission mitigation pledges: Metrics and institutions' in Scott Barrett, Carlo Carraro, and Jaime de Melo (eds) Towards a Workable and Effective Climate Regime (VoxEU.org Books, 2015) 167-181; Joseph E Aldy, 'Evaluating Mitigation Effort: Tools and Institutions for Assessing Nationally Determined Contributions' (2015) Discussion Paper: Harvard Project on Climate Agreements <http://pubdocs.worldbank.org/en/736371454449389076/pdf/ Evaluating-Mitigation-Effort-Nov-2015.pdf $>$ accessed 27 February 2018; Joseph E Aldy and William A Pizer, 'Alternative Metrics for Comparing Domestic Climate Change Mitigation Efforts and the Emerging International Climate Policy Architecture' (2016) 10 Review of Environmental Economics and Policy 1; Joseph E Aldy, William A Pizer \& Keigo Akimoto 'Comparing emissions mitigation efforts across countries' (2017) 17 Climate Policy 4, 501-515.

22 Joseph E Aldy, 'Evaluating Mitigation Effort: Tools and Institutions for Assessing Nationally Determined Contributions' (2015) Discussion Paper: Harvard Project on Climate Agreements <http:// pubdocs.worldbank.org/en/736371454449389076/pdf/Evaluating -Mitigation-Effort-Nov-2015.pdf> accessed 27 February 2018

23 As to exchange rates, see also: Lazarus et al, 'Options and Issues for Restricted Linking of Emissions Trading Systems', (ICAP, September 2015) <https://icapcarbonaction.com/en/?option=com _attach\&task=download\&id=279> accessed 6 September 2016 .

24 Michael Mehling, 'Legal Frameworks for Linking National Emissions Trading Schemes', in Carlarne, Gray and Tarasofsky (eds), Oxford Handbook of International Climate Change Law, (OUP, 2016) 276 .

25 (n 22) 13 
on transparency and effective review mechanisms, to give credibility to commitments, the idea of transparency and policy surveillance of countries in the context of multilateral regimes, not being something new. ${ }^{26}$ A number of transparency models from other multilateral regimes can be cited, including the International Monetary Fund (IMF) annual countrylevel economic surveillance; the Organisation for Economic Cooperation and Development (OECD) peer reviews of member states' economic policies every one or two years; and the World Trade Organisation (WTO) regular reviews of members' trade policies. The conclusion is that the international community can draw on an array of transparency and policy surveillance models. ${ }^{27}$

Further, Aldy draws on economic game theory, international relations and legal academic literature to support the conclusion that policy transparency and surveillance can generate better outcomes in climate change negotiations, whether that be through legitimising a participant country's domestic policies, limiting the capacity for free-riding, or reducing overall costs of information gathering and so levelling the playing field. ${ }^{28}$ While the context being considered in this paper differs, in that it is not climate change negotiations per se, but rather the mechanism that might be applied to give effect to the outcome of those negotiations, namely the market in which mitigation outcomes are traded, Aldy's arguments apply equally to the assessment of MV as part of a better design for effecting transfers of mitigation outcomes.

To this, one can add the fact that countries undergo sovereign credit rating assessments in order to borrow. For example, Standard \& Poor's sovereign issuer credit ratings, pertaining to a sovereign's abili-

\footnotetext{
26 ibid.

27 ibid 34.

28 Joseph E Aldy, 'Designing a Bretton Woods Institution to Address Climate Change' (2012) HKS Faculty Research Working Paper Series RWP12-017

29 S\&P Global Ratings, 'Sovereign Rating Methodology' (2017) <https://www.spratings.com/documents/20184/4432051/ Sovereign+Rating+Methodology/5f8c852c-108d-46d2-add1 -4c20c3304725> accessed 1 March 2018.

30 Additionally, under one model proposed, countries might even do so on their own terms. See, Justin D Macinante, 'A Conceptual Model for Networking of Carbon Markets on Distributed Ledger Technology Architecture' (2017) 3 CCLR, 243-260.

31 ibid

32 ibid.

33 (n 21)
}

ty and willingness to service financial obligations to commercial creditors, encompasses a framework including (amongst other factors) policymaking; income levels, GDP per capita, tax and funding bases; currency in international transactions, external liquidity, residents' assets and liabilities relative to rest of the world; sustainability of debt burden; and exchange rate regime and monetary policy credibility. ${ }^{29}$ Countries' sensitivities to disclosure of these sorts of statistics seem to recede when the objective at stake is access to international debt markets. Why should there be a difference when it comes to accessing an international carbon market, for which there may be similar financial, trade and policy benefits (to those mentioned by Aldy above) $?^{30}$

The second line of argument is that many, if not most, of the sources of potential political controversy can be addressed through careful regime design. For instance, consider the conceptual model for networking carbon markets on a distributed ledger architecture, proposed by the author in an earlier paper. ${ }^{31}$ It is postulated that elements of that model, were such a regime to be given effect, ameliorate the causes of potential political controversy, for instance: - by ensuring the independence of the process to quantify and compare mitigation effort and that the entity or entities carrying out that assessment comprise relevantly qualified, independent, impartial experts;

- by applying generic criteria to assessments uniformly across all jurisdictions in that process, such that all jurisdictions are subject to equivalent treatment under the process;

- ensuring that the process and outcome are open and transparent and that outcomes are communicated appropriately as market sensitive information; and

- affording all jurisdictions the flexibility to engage with, or leave, the process relatively easily and on the same basis - in the event that, as an information tool, the assessment is part of an agreed governance framework (as opposed to being purely private sector driven). ${ }^{32}$

Aldy argues that transparency and policy surveillance in international relations are not only beneficial, but accepted already in a number of contexts. Aldy also considers principles for developing effective metrics for mitigation effort and what those metrics might be. ${ }^{33}$ The following sub-section of this pa- 
per now reviews those and some other specific approaches that are developing, or could be adapted into, methodologies for determining the MV of mitigation outcomes.

\section{Methodologies}

Mitigation outcomes will be measurable and notwithstanding some being absolute while others are relative, or intensity-based as opposed to emission-based, it is presumed that all measured outcomes will be capable of being converted into the same empirical metric, such as tonnes of $\mathrm{CO}_{2}-\mathrm{e}$, per unit, per period of time (e.g., say, per annum). As noted above, to enable comparability, adjustments need to be made to these measured amounts to take account of differences in the contexts in which the outcomes are achieved. The adjustments might be based on generic parameters relating to:

- the mitigation action itself (such as concerning risk factors associated with the action, its monitoring, measurement, reporting, or verification);

- the suite of actions of which it forms part in the particular jurisdiction where it is carried out (for example, the overall percentage of jurisdictional emissions covered);

- the jurisdiction itself (for instance, economic size, production, economic structure, level of emissions, financing considerations, level of ambition, capacity). ${ }^{34}$

These parameters illustrate an approach to determining MV. The measured value or outcome, so adjusted, would be the MV of the unit of 'carbon asset' produced by the mitigation action considered. Similar assessment methodologies or processes include the NCM's Mitigation Action Assessment Protocol (MAAP) trialled by the World Bank's Partnership for Market Readiness, the Climate Transparency approach, and the more academic approach to deriving suitable metrics taken by Aldy and colleagues.

\section{a. World Bank NCM Initiative: MAAP}

The MAAP ${ }^{35}$ differs from the context of transfers between ETSs described above, in that to date it has focused on the MV of project-generated credits. 'In the long run, the MAAP is intended to contribute to achieving the goal of an independent and interna- tionally accepted system for comparing carbon assets and eventually, trade and exchangeability of carbon credits. ${ }^{36}$ Nevertheless, the approach and to some degree, the metrics and methodology, could be applicable not just to carbon credits, but also to other types of mitigation actions.

The MAAP is continuing to evolve, currently consisting of four independent risk modules, the first three of which relate to environmental (carbon) integrity, while the fourth covers developmental benefits. To these a fifth module, relating to international transfer readiness under Article 6.2 of the Paris Agreement, is added. The first three modules are: mitigation action program; mitigation action management entity; and investment environment. Each module is comprised of assessment areas for which there are key indicators. So, for instance, the mitigation action program module has assessment areas: definition \& scope; objective \& targets; planning; roles, responsibilities and authorities; barriers; emissions reduction from intervention; and monitoring and reporting. Each assessment area is weighted according to its relevance and the key indicators in each are also weighted according to the level of confidence for that indicator. ${ }^{37}$

While the first module (mitigation action program) includes 'emission reductions from interventions' as one of the seven assessment areas, with seven of the thirty-two indicators, its relative weight in the scoring is only $20 \%$, suggesting emphasis on overall design, governance and planning, rather than on physical mitigation measured. For example, definition and scope $(14 \%)$ and objectives and targets $(20 \%)$ have a higher overall weighting than emission reductions $(20 \%)$ and monitoring and reporting $(10 \%) .^{38}$ This emphasis may reflect the short-term ob-

34 This third adjustment factor, for instance, inter alia, would take account of the elements in Arts 2.2 and 4.3 Paris Agreement, namely common but differentiated responsibilities, respective capabilities, and different national circumstances.

35 World Bank Partnership for Market Readiness, 'Mitigation Action Assessment Protocol' (April 2016) 110153-WP-P161139-PUBLICMAAPMay <http://www.worldbank.org/en/topic/climatechange/ brief/globally-networked-carbon-markets $>$ accessed 27 Februrary 2018.

36 ibid 10

37 ibid 19-22, an illustrative table of indicators and weightings is set out. Modules are also set out in Annex 1.

38 They also have nine indicators as opposed to ten, suggesting the average level of confidence in the indicators for the latter two would be $10 \%$ less, although it is noted that these are all just illustrative. 
jectives of MAAP to help jurisdictions at the program level to systematically self-evaluate their mitigation actions and demonstrate the results. Hence, guidance on design and implementation, and prioritisation of actions in climate change strategic planning are to the fore.

The recently added fifth module assesses sector/jurisdictional readiness to engage in Article 6.2. As presently conceived, it covers four equally weighted assessment areas, being: double counting; environmental integrity; alignment of mitigation outcomes with NDC; and transparency. Each of these has a number of key indicators, which again are allocated different weightings. One issue that will need to be guarded against in relation to these is the risk of overlap, given the conceptual closeness of the assessment areas.

The MAAP modular scoring approach can be contrasted with that of a second initiative, also supported by the World Bank, promoted by Climate Transparency.

\section{b. Climate Transparency}

By focusing on projects, MAAP might be considered to be the micro-level, while the approach taken by Climate Transparency is at the opposite, macro-level end of the spectrum. Climate Transparency prepares the annual 'Brown to Green' report examining G2O countries' transition to a low-carbon economy, which collates a range of indicators including emissions and emission trends, climate policy performance, indicators on how countries are financing the transition, and indicators relating to how they are decarbonising. ${ }^{39}$

The indicators are garnered from a number of sources including the World Bank, International Energy Agency (IEA), Organisation for Economic Cooperation and Development (OECD), various United Nations agencies, non-governmental organisations

39 See, Climate Transparency, 'Brown to Green: The G20 Transition to a Low-Carbon Economy' (2017) <https://germanwatch.org/ sites/germanwatch.org/files/publication/18761.pdf> accessed 12 August 2018.

40 Author's private communication with Climate Transparency, 1 June 2018.

41 ibid.

42 Joseph E Aldy and William A Pizer, 'Comparing emission mitigation pledges: Metrics and institutions' in Scott Barrett, Carlo Carraro, and Jaime de Melo (eds) Towards a Workable and Effective Climate Regime (VoxEU.org Books, 2015) 167-181. such as Germanwatch, Climate Action Tracker and private sector sources. It is interesting that Climate Transparency is a partnership with organisations from Argentina, Brazil, China, France, Germany, India, Indonesia, Mexico, South Africa and UK. The report is put together by the partnership with the intention of achieving a wider perspective and therefore hopefully deriving a broader legitimacy. ${ }^{40}$

The Brown to Green reports rank G2O countries in relation to the individual indicators and provide overviews individually for each country. While these individual indicators allow comparison on a disaggregated basis, the overviews are descriptive and the reports do not aggregate the indicators to give a single overall figure. At present, Climate Transparency deliberately abstains from aggregating the indicators so as to provide comparable information to governments. An index, created from aggregating indicators (as, for example, is done by Germanwatch, one of the Climate Transparency partners) has a better media communication appeal but does not necessarily promote meaningful interaction with governments. ${ }^{41}$

Thus, while the individual Climate Transparency indicators may provide valuable input to an MV assessment methodology, the reports overall do not promote a methodological approach for such. Both the MAAP and Climate Transparency approaches include indicators that might be applicable and feed into a methodology to assess MV, yet neither approach individually, as it currently stands, could provide MV assessment.

\section{c. Aldy and Colleagues}

As noted earlier, Aldy and his colleagues have considered principles for developing effective metrics for mitigation effort. In looking at metrics for comparing emission mitigation pledges, Aldy and Pizer ${ }^{42}$ identify three principles to help inform the selection as comprehensiveness; measurability and replicability; and universality. The metrics they consider fall into three categories:

- emissions and other physical measures (that have direct relevance to the environment);

- prices on carbon and energy taxes (reflecting policies to reduce emissions); and

- costs (that measure the useful economic resources diverted away from consumption and towards abatement). 
These authors note that, in practice, there will be trade-offs in the degree to which any particular metric satisfies the principles. ${ }^{43}$ For instance, emission levels, usually expressed relative to a baseline year, were applied under the Kyoto Protocol. Apart from issues over what baseline year might be set, emissions do not necessarily reflect effort, as evidenced by the issues over hot air from economies in transition. Emission intensity is also problematic, in that it can be influenced by changes both in economic development and technology uptake. It is noted that observed emissions might be compared to an analysis of what emissions would have been without mitigation policies, in effect, a retrospective forecast, to give a comprehensive metric. ${ }^{44}$ Carbon prices, energy taxes and economic costs similarly, all have drawbacks. As such, a portfolio of metrics along the lines of the suite of economic statistics used to describe the health of the macroeconomy is recommended. ${ }^{45}$

These principles and metrics are analysed further in a second paper looking at alternative metrics for comparing mitigation effort. ${ }^{46}$ The analysis reveals that metrics such as total emissions and explicit emission prices are easy to observe and measure, but may be removed from key concepts of effort and underlying policy implementation. Metrics that more closely reflect effort, such as emission reductions, implicit prices and costs, are harder to observe and measure, introducing subjectivity and possibly inconsistency. Metrics can be constructed or benchmarked in a variety of ways that may or may not adjust for endowments, historical behaviour or future growth. The authors conclude that more appropriate relative comparisons could be achieved by grouping countries with peers. ${ }^{47}$

Aldy develops this further in terms of the need for evaluation and assessment tools generated by the significant heterogeneity of approaches apparent in the Paris Agreement pledge and review paradigm. ${ }^{48}$ The key point is the need for economic modelling tools and sophisticated analysis, to inform decision making, not least to account for competitiveness issues for business stakeholders, as well as underscoring the fairness for countries of multilateral arrangements. Assessment and comparison of mitigation effort can also highlight similarities or differences in expected marginal abatement costs, leading to great potential efficiencies. ${ }^{49}$

A template framework of metrics of efforts and comparison of countries is presented in a more re- cent paper. ${ }^{50}$ Building on the earlier work, the intention is to provide a common data and analytic framework that can measure mitigation efforts and facilitate like-for-like comparisons. The metrics are again in terms of: (i) emissions corresponding to physical outcome measures; (ii) prices on carbon and energy reflecting market signals designed through mitigation policies; and (iii) cost metrics including measures of economic resources diverted away from current consumption.

Viewed alone, none of the metrics does well in terms of the principles (comprehensiveness; measurability and replicability; and universality) against which they are evaluated. Hence a suite of metrics is proposed to compare effort and a template framework presented. ${ }^{51}$ Approaches for determining or benchmarking whether country efforts are 'fair' or 'satisfactory' are considered, such as, their ranking against each quantitative metric (this is similar to the Climate Transparency approach, but would not indicate whether or not an effort was satisfactory or fair), or arranging countries into peer groups for relative comparisons (would identify laggards), or establishing absolute benchmarks (for example, as negotiated under the Kyoto Protocol, but unlikely under pledge and review).

Yet, these authors admit, any attempt to carry out such an exercise would need to account for issues such as population growth, wealth, fossil fuel resources, as well as judgments about past actions and the weight to be placed on new efforts. They conclude that despite the increasing relevance of metrics and

43 ibid.

44 This is, in effect, what is being described in Section II of this paper as the primary mitigation value, to which the adjustments based on risk need to be made.

45 (n 42).

46 Joseph E Aldy and William A Pizer, 'Alternative Metrics for Comparing Domestic Climate Change Mitigation Efforts and the Emerging International Climate Policy Architecture' (2016) 10 Review of Environmental Economics and Policy 1.

47 ibid 27.

48 Joseph E Aldy, 'Evaluating Mitigation Effort: Tools and Institutions for Assessing Nationally Determined Contributions' (2015) Discussion Paper: Harvard Project on Climate Agreements <http:// pubdocs.worldbank.org/en/736371454449389076/pdf/Evaluating -Mitigation-Effort-Nov-2015.pdf> accessed 27 February 2018. 49 ibid.

50 Aldy, Joseph E., William A. Pizer and Keigo Akimoto (2017) Comparing emission mitigation efforts across countries, Climate Policy, 17:4, 501-515.

51 ibid Table 1. 
analytical tools to compare mitigation actions across countries, it is unlikely that multilateral negotiations will reach a consensus on this in the near term such that, in the meantime, independent researchers and expert analysts will need to fill the gap.

\section{d. Other Approaches and Developments}

\section{i. Standards Platforms, e.g., Gold Standard and Others}

While standards considered in this subsection apply to project generated credits, rather than allowances under ETSs, they illustrate another approach that has been taken to the question of valuing mitigation actions. Under the Kyoto Protocol modalities and procedures for the clean development mechanism, a certified emission reduction (CER) is defined to be 'a unit issued pursuant to the relevant provisions in the annex to decision-/CMP.1 (Modalities for the accounting of assigned amounts) and is equal to one metric tonne of carbon dioxide equivalent, calculated using global warming potentials defined by decision 2/CP.3 or as subsequently revised in accordance with Article 5. ${ }^{52}$ Consequently, for a CDM project, the CDMEB will either issue CERs each of that value, or not issue them, depending on its assessment of the project. Thus it is a binary process, with no scope for levels of mitigation value.

Consequently, standards platforms such as the Gold Standard (GS), Voluntary Carbon Standard (VCS) and others, ${ }^{53}$ which apply to project-generat-

52 Report of the Conference of the Parties on its Seventh Session, held at Marrakesh from 29 October to 10 November 2001, FCCC/CP/2001/13/Add.2 21 January 2002, Decision 17/CP.7, 26.

53 Seven such standards were reviewed in 2008 by WWF: Anja Kollmuss, Helge Zink, Clifford Polycarp (WWF Germany), 'Making Sense of the Voluntary Carbon Market A Comparison of Carbon Offset Standards (2008) < http://www.globalcarbonproject .org/global/pdf/WWF_2008_A\%20comparison\%20of\%20C $\% 20$ offset\%20Standards.pdf $>$ accessed 3 June 2018.

54 This is the market in which businesses, individuals and other entities that are not subject to any legal requirements to do so, nevertheless voluntarily purchase and cancel credits so as to offset their carbon emissions. Some GS CERs have been used for compliance.

55 (n 53) 88-93

56 It is noted also that these VERs are sold in different voluntary markets to a variety of different purchasers, whose motivations may also vary as to what factors are important.

57 KLIK, 'Kompensation im Ausland nach den Regeln des Pariser Übereinkommens' <https://www.international.klik.ch/en/ Regulatory-framework.214.html> accessed 4 June 2018.

58 ibid ed credits but operate mostly in the voluntary market, ${ }^{54}$ also work on the basis that the credits they certify are of a value of one tonne $\mathrm{CO}_{2}$-e. This is in spite of the fact that, not only can the projects themselves vary considerably in terms of factors such as the source of reductions, methodology, process, monitoring, reporting and verification, but the standards applied differ in terms of accounting, monitoring, verification and certification, and review. For instance, a WWF report identified differences in standards reviewed for additionality tests, third party verification requirements, separation of verification and approval process, whether the standard had a registry, accepted project types, whether project types with a high chance of adverse impacts were excluded, and whether account was taken of potential co-benefits. ${ }^{55}$ The credits certified under the different standards range in price from GS CERs that can trade at a premium to CERs, down to credits for as little as EUR 1, although being the voluntary market, different factors affect demand than in the case of the compliance market. ${ }^{56}$ Nonetheless, it seems incongruous that in spite of the differences, purchasers who buy these credits will be offsetting them against their emissions all on the basis that they equal one tonne $\mathrm{CO}_{2}$-e.

The impact of MV assessment applied to projectgenerated credits is potentially twofold (at least) for these standards: first, just as for allowances from heterogeneous schemes, it will facilitate fungibility of the credits; and secondly, it will shine a bright light on the standards themselves, exposing them to closer scrutiny which will benefit the more rigorous, but perhaps cause difficulties for those that are less so.

\section{e. Market Purchasers, e.g., Klik Foundation in Switzerland}

Organisations may be active in the carbon markets for a variety of reasons. For instance, the Foundation for Climate Protection and Carbon Offset (KliK) is a sector-wide carbon offset grouping for fossil motor fuels, under Swiss $\mathrm{CO}_{2}$ law. ${ }^{57}$ In Switzerland, mineral oil companies responsible for releasing fossil motor fuels for consumption must offset part of the emissions resulting from use of these fuels, which the KliK Foundation does on their behalf. ${ }^{58}$ While the Swiss law is under revision (in the context of the anticipated guidance on Article 6), draft proposals include that the KliK Foundation should look to the in- 
ternational market for reductions (presumably mitigation outcomes) that will help Switzerland meet its NDC. How these mitigation outcomes are valued is thus of critical importance. Future market participants such as KliK Foundation will need to consider the criteria they apply in making such purchases, in the absence of a recognised system for MV assessment.

\section{f. Further Possibilities}

There are many potential variations on the methodological approach to MV canvassed in this paper. For instance, one might involve combining the MAAP and Climate Transparency indicators. ${ }^{59}$ Another illustrative approach is one that anchors mitigation value to the policy objective of the UNFCCC by valuing the ambition expressed by Parties to the Paris Agreement through their NDCs: by starting from the level at which global emissions must be capped to enable temperature increase to be limited to $1.5^{\circ} \mathrm{C}$, a comparison can be made with the aggregate commitments of Parties as expressed through their NDCs. Consideration of whether the collective ambition of the NDCs is consistent with the $1.5^{\circ} \mathrm{C}$ limit, of what individual Parties' fair burden sharing contributions might be, and of whether those Parties will actually comply with their committed levels of emissions, can provide a mitigation value. ${ }^{60}$ This MV might be operationalized through a discount factor in terms of the global temperature goal for carbon asset units and an exchange rate based on the relative ambition of the transaction participants. The discount factor and exchange rate would be applied to exported units, which would need to be 'budget compliant'. ${ }^{\prime 1}$ This approach also invites exploration of the extensive literature on methodologies for determining equitable distribution of mitigation targets, which while relevant to consideration of the third of the risk factors mentioned earlier (also at (c) in the following, concluding section), are not considered in this paper. $^{62}$

\section{Conclusion on Methodologies}

In terms of an emissions trading scheme (ETS), as illustrated in Section II, the starting point for MV assessment is the difference in emissions with and without the scheme: that is, the projected or observed emissions under the ETS (depending on design) compared to an analysis of what emissions would have been without the ETS, in effect, a retrospective forecast. This represents the (projected) amount of emissions reduced by the ETS in the relevant time period, if entities under that scheme have complied. ${ }^{63}$ While Aldy describes such an approach as 'a comprehensive metric', to more accurately reflect what can be described as the value of the measured outcome, adjustments need to be made to that amount to take account of differences in the contexts in which the outcomes are achieved. To recap, adjustments proposed might be based on parameters relating to:

a) the mitigation action itself (such as concerning risk factors associated with the action, its monitoring, measurement, reporting, or verification, reliability of the BAU forecast);

b) the suite of actions of which it forms part in the particular jurisdiction where it is carried out (for example, the overall percentage of jurisdictional emissions covered);

c) the jurisdiction itself (for instance, economic size, production, economic structure, level of emissions, financing considerations, level of ambition, capacity).

The first of these categories can be seen to parallel the MAAP approach, while the second and third are similar to Climate Transparency, the MAAP fifth module and the metrics identified by Aldy and his colleagues, whose principles for selecting appropriate metrics also provide guidance.

Yet, as these commentators identify also, despite the increasing relevance of metrics and analytical tools to compare mitigation actions across countries, it is unlikely that multilateral negotiations will reach

59 The author understands that research on such an approach may be presently under consideration by the World Bank.

60 Johannes Heister, 'Mitigation Value to Enable International Linkage of Domestic Programs' (Networked Carbon Markets initiative strategy workshop, Cologne, 28 May 2016)

61 Justin Macinante, 'Networking Carbon Markets: Key Elements of the Process' (2016) $18<$ https://openknowledge.worldbank.org/ handle/10986/25750> accessed 21 August 2017.

62 For a recent analysis, in context of the fairness of Australia's NDC target, see, Merzian et al, 'Advance Australia's fair share, Assessing the fairness of emissions targets' (12 June 2018) The Australia Institute, 22 <http://www.tai.org.au/sites/defualt/files/P507 $\% 20$ Advance $\% 20$ Australias\%20Fair\%20Share\%20FINAL_0.PDF> accessed 12 June 2018.

63 As noted earlier, the marginal effect may be a more appropriate starting point. 
a consensus on this soon. The recent SBSTA48 negotiations appear to bear out this expectation. As such, the field remains open for independent researchers and expert analysts to fill the gap in devising a methodology or methodologies for assessing MV, enabling fungibility of units from different mitigation actions and thereby facilitating transfers of the outcomes of those actions. In short, there is an opportunity for the private sector to step in and fill the current void.

\section{Institutional Arrangements for Overseeing Mitigation Value Assessment}

Notwithstanding the conclusion of the preceding section, there will be a need for institutional arrangements to oversee any process for assessing mitigation value that may gain acceptance. While there may be others, by way of illustration, two alternative possibilities for how institutional arrangements might develop canvassed by this paper are:

- firstly, by a public, intergovernmental institution, possibly along the lines of the Clean Development Mechanism Executive Board (CDMEB) model;

- secondly, by private sector entities, under a model similar to that which operates for credit reference/rating agencies (CRAs), subject to a regulatory model such as that administered by the European Securities and Markets Authority (ESMA).

\section{Public, Intergovernmental Institution Model}

The public, intergovernmental institution model is perhaps more problematic, in terms of fostering re-

\footnotetext{
64 For the a view on the dependence of this market on investor confidence see, Charlotte Streck and Jolene Lin 'Making Markets Work: A Review of CDM Performance and the Need for Reform' (2008) 19 European Journal of International Law 2, 420.

65 ibid; Ilona Millar, Martijn Wilder, 'Enhanced Governance and Dispute Resolution for the CDM' (2009) CCLR 45 (2009). Recommendations for how the issues can be rectified are noted, as are the alternative models raised by these authors.

66 (n 64).

67 Joseph E Aldy, 'Designing a Bretton Woods Institution to Address Climate Change' (2012) HKS Faculty Research Working Paper Series RWP12-017.

68 ibid 13 et seq.
}

engagement in the market by the private financial sector, given aspects of the CDMEB experience. ${ }^{64}$ Is- $^{-}$ sues raised in relation to CDMEB operations have included a lack of transparency (in spite of provisions in its rules for public disclosure), lack of clarity and predictability in decision-making, and the absence of decision review or appeal rights. Its nature as a body made up of regional negotiating group nominees, rather than a panel of independently assessed, expert appointees, has been flagged as well. ${ }^{65}$ Further, the CDM process has been lengthy and cumbersome. ${ }^{66}$ The complexity of the CDMEB's role, including as de facto gatekeeper over the flow of projects to the market has been problematic. The lesson from this for the MV process is that any structure needs to separate the function of regulating providers of $\mathrm{MV}$, from the actual provision of $\mathrm{MV}$, which should just be market information, available openly to, and independently of, market operation.

Another perspective is that taken by Aldy, ${ }^{67}$ who in 2012 proposed the design of a Bretton Woods Climate institution, to enhance public knowledge about nations' commitments, policies and outcomes. Such an institution would have three primary functions: to implement a system of national and global policy surveillance; to promote best practice policies; and to provide a means to channel financing for investments in climate change risk mitigation activities in developing countries. ${ }^{68}$ The experiences of other international policy regimes are drawn upon to provide lessons that might inform the design of such a body, for instance, reports compiled after International Monetary Fund (IMF) country visits, which form the basis for peer review by the Executive Board of country directors, and ultimately are published so as to enable stakeholder engagement. Similarly, Aldy mentions Organisation for Economic Co-operation and Development (OECD) economic surveys, which also have a focus on peer review.

In synthesising the other regimes reviewed, Aldy notes that: first, the other regimes rely on both expert review and peer review, whereas the climate regime only has expert review; secondly, the expert reviews under other regimes are undertaken by career staff of their respective institutions, while the climate regime typically relies in an ad hoc fashion on government-sponsored experts; thirdly, there is a distinction under the climate regime between industrialised and other states (although this is changing under the Paris Agreement); and fourthly, standards 
and reporting templates improve transparency of reporting and review, enhancing surveillance effectiveness. $^{69}$

These points of distinction between the climate and other regimes and the lessons identified by Aldy are important. However, they are observed in a context that differs from that in which the MV assessment will be most relevant, namely the operationalization of Article 6 Paris Agreement through transfers of mitigation outcomes: trading carbon assets between heterogeneous schemes. In this context, additional considerations need to be taken into account, for instance, the confidence of the market in the accuracy and reliability of the MV assessment process; and the capacity of that process to adjust to or account for the dynamic nature of the information on which it is based, or the possibility of the need to rectify inaccurate assessments. These considerations raise the question of how flexible a public, intergovernmental institution might need to be, and whether such a model could achieve the level of flexibility and reliability that might be required to meet the demands of an efficiently operating market.

\section{Regulated Private Sector Model}

The second approach would entail private sector (CRA-type) entities being accredited to assess and determine MVs, based on approved methodologies, subject to authorisation and supervision along the lines of the ESMA regulatory model for CRAs. The outcomes would be publicly available market information. Detailed consideration has been given to this approach in an earlier World Bank paper, ${ }^{70}$ the relevant sections of which are transposed and adapted in an Annex available online. ${ }^{71}$

Reasons why this approach might be favoured over the public, intergovernmental institution model include, first, its greater flexibility. As noted above, the nature of the information taken into account for the purpose of assessing MV in the case of any jurisdiction will not be static. It will be constantly changing, with new information that comes to hand needing to be factored into calculations. Some such updated information may be less immediately critical to assessments, while other revisions may necessitate changes on a more dynamic basis. With multiple private sector institutions, rather than a single public one, employing more assessors making the as- sessments, it is conceivable that better accounts might be taken of these informational change requirements.

Second, by having a greater number of entities providing MV assessments there would be scope for greater statistical reliability in the process. More entities providing MV assessments would open the way for statistical analysis to identify and exclude outliers, thereby reducing the potential impact significance of unavoidable value judgments and subjective criteria that inevitably will be implicit in any methodology.

Third, the regulated private sector model proposed here is one with which the private financial sector will be more familiar, based on the parallels with the CRA process. Thus, the hope would be that such familiarity would translate into readier acceptance of the process.

As to whether, or how, the necessary consensus amongst Parties might be secured in order that a governance framework for accreditation might be established, that remains an unanswered question at this point in time. Nevertheless, it remains open for the private sector to grasp the initiative and introduce MV assessment services.

\section{Conclusion}

Emissions level growth is increasing and without additional efforts will persist, driving global mean temperatures to unmanageable levels. ${ }^{72}$ To achieve the climate change policy objective of limiting the increase to less than $2^{\circ} \mathrm{C}$, there is a limited budget for cumulative global GHG emissions: at the current rate of emissions, that budget will be exceeded in less than twenty years. $^{73}$

Efforts to bring about the additional mitigation are expanding across jurisdictions globally, often involv-

69 ibid.

70 (n 61).

71 Justin Macinante, 'Operationalizing Cooperative Approaches Under the Paris Agreement by Valuing Mitigation Outcomes' (2018) Available at SSRN <http://ssrn.com/abstract=3211454>

72 IPCC, 2014: Summary for Policymakers. In: Climate Change 2014: Mitigation of Climate Change. Contribution of Working Group III to the Fifth Assessment Report of the Intergovernmental Panel on Climate Change. Cambridge University Press, Cambridge, United Kingdom, 6 .

73 (n 62) 22. 
ing mechanisms to put a price on carbon. These measures, while reflecting local circumstances, are diverse and fragmented. To be successful in bringing about behavioural changes to increase mitigation of GHG emissions, they need to engage the private sector at scale. To do so, there need to be well-designed, properly functioning markets for the international transfer of mitigation outcomes between these diverse jurisdictional schemes. Assessment of mitigation value provides the means to enable comparability between, and fungibility of units across, those schemes so that the desired scale might be achievable.

Private sector entities, researchers and analysts should be encouraged to step in and fill the void that exists in terms of methodologies for assessing mitigation value. Concurrently, consideration should be

74 BBC, 'Trump White House axes Nasa research into greenhouse gas cuts' (BBC News Online, 10 May 2018) <https://www.bbc .com/news/world-us-canada-44067797> accessed 12 August 2018. given at the intergovernmental level to appropriate institutional arrangements that might be put in place to guide, manage and oversee such methodological processes.

At the same time as the inconclusive SBSTA48 negotiations in Bonn were closed, it was reported that the Trump Administration was ending funding for NASA's monitoring system for greenhouse gases. ${ }^{74}$ The loss would jeopardise the ability to measure national emission cuts, it was reported, weakening the chances of developing a robust and transparent emission monitoring system.

In these unfortunate circumstances, surely it is important for the piloting of models that offer the potential for effectively operationalizing the Paris Agreement to commence without delay, rather than waiting for interminable negotiations to resolve every process-issue dispute. In the meantime, GHG emissions are continuing to rise, to a certain extent unmeasured and unmonitored, and the manifestations of a changing climate continue to become ever more obvious, more frequent and more damaging. 